\title{
Détermination des caractéristiques thermiques des roches anisotropes par une méthode de choc thermique
}

\author{
Determination of anisotropic rocks thermal properties \\ by a thermal shock method
}

K. SU, Ph. WEBER

Ecole des Mines d'Alès ${ }^{\circ}$

Rev. Franç. Géotech. n 55 , pp. $63-74$ (avril 1991)

\section{Résumé}

Les travaux actuellement développés à l'Institut des Matériaux et des Gisements Miniers de l'Ecole des Mines d'Alès sont relatifs à l'étude des propriétés de transfert thermique dans les roches anisotropes. Une méthode de choc thermique (dite du "fil chaud ») est mise en œuvre pour déterminer les paramètres thermiques des roches anisotropes : conductivités et chaleur spécifique. Nos études montrent que cette méthode peut être adaptée aux milieux anisotropes, notamment les roches. Nous présentons la technique utilisée, les analyses théoriques et les premiers résultats.

\footnotetext{
Abstract

The Mining School of Alès (France) is developping a reseach program concerning thermal properties of rocks. This paper presents an application of thermal shock methods to anisotropic rocks. This method leads to simultaneous determination of both specific heat and principal thermal conductivities; it allows to perform experiments with specimens at hight ambiant temperature and pression. A description of experimentation is given and the first results are pointed out.
} 


\section{DESCRIPTION DE LA MÉTHODE}

Connue depuis de nombreuses années, la méthode du choc thermique consiste à utiliser une résistance chauffante (sonde) dans laquelle passe un courant dissipant une certaine puissance. L'évolution de la température d'un point du matériau est fonction de la puissance électrique dissipée, et des propriétés thermiques du matériau. Plusieurs travaux ont été consacrés aux différentes procédures d'identification des paramètres.

PERRIN et al. (1980, 1982) [9, 13] présentent, dans plusieurs publications, la méthode de mesure de conductivité en utilisant les températures à la sonde, et celle de la diffusivité par l'analyse du thermogramme (temps de montée en température, valeur de la température maximale). AUDIBERT (1985) [1] et LAURENT (1986) [11] fournissent des études d'optimisation d'outils pour déterminer les caractéristiques thermocinétiques de milieux poreux en adaptant le modèle de la sonde cylindrique. Un outil dit "tritige » (deux tiges pour les capteurs de température au sein du milieu et une tige pour la sonde) semble bien adapté pour la mesure in situ.

Pour les roches, une difficulté particulière est liée à la mise en place de la sonde électrique et des thermocouples, dans des trous de faible diamètre et de longueur importante. CULL et al. (1974) [6] proposent de scier un bloc de roche en deux parties et d'y tailler ensuite une petite "gorge " dans laquelle la sonde est logée ; les auteurs ont mesuré la conductivité d'après le thermogramme de la sonde dont le diamètre est considéré comme infiniment mince; pour les roches anisotropes, l'analyse d'un seul thermogramme ne permet pas d'identifier les deux conductivités principales. Nos études montrent que les mesures de température doivent s'effectuer en au moins deux points du milieu.

En conséquence, l'échantillon est découpé suivant un plan de symétrie de la structure pour y placer la sonde, (fig. 1) : en supposant que le flux de chaleur se diffuse perpendiculairement à l'axe de la sonde (écoulement plan), l'existence de l'interface n'a donc théoriquement pas d'influence sur le transfert de chaleur.

Nous proposons également, si la roche est très dure, de scier l'échantillon en plusieurs plaques, (fig. 2), puis de percer des trous traversant les plaques; les points de mesure sont situés sur la plaque centrale dans des trous de faible profondeur; lavantage de cette méthode réside dans le fait que la position du thermocouple peut être repérée avec précision. Nous supposons, dans cette configuration d'essai, que le transfert thermique s'effectue toujours perpendiculairement à l'axe de la sonde; les interfaces entre les plaques parallèles aux plans de diffusion de chaleur n'ont pas d'influence sur le transfert de la chaleur (gradient de température nul parallèlement à l'axe de la sonde).

La figure 3 illustre l'ensemble du dispositif ; les températures sont mesurées à l'aide de thermocouples dont les soudures chaudes sont mises dans les trous avec une colle spéciale. Les signaux sont amplifiés par les transmetteurs de thermocouple reliés à une carte

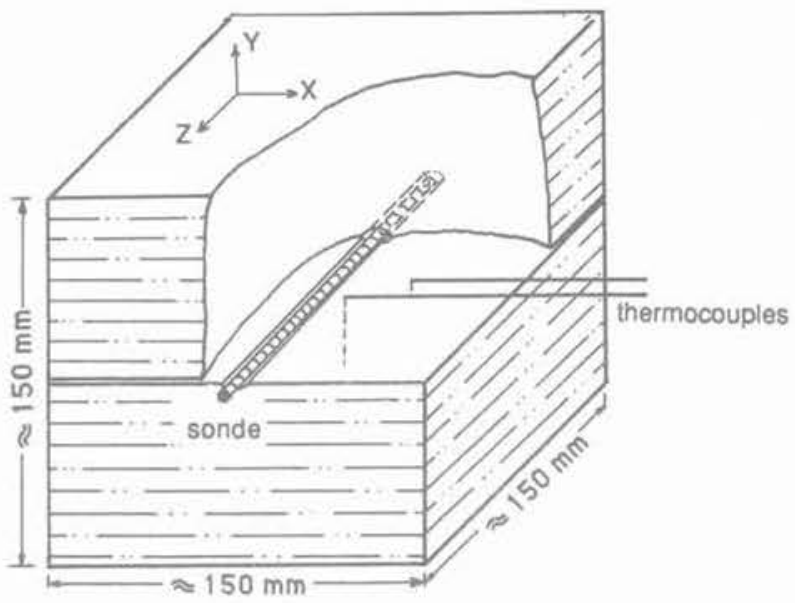

Fig. 1. - Schéma de l'expérience.

(lci, le bloc est scié en deux parties suivant le plan horizontal passant par l'axe de la résistance chauffante). Fig. 1. - View of the sample.

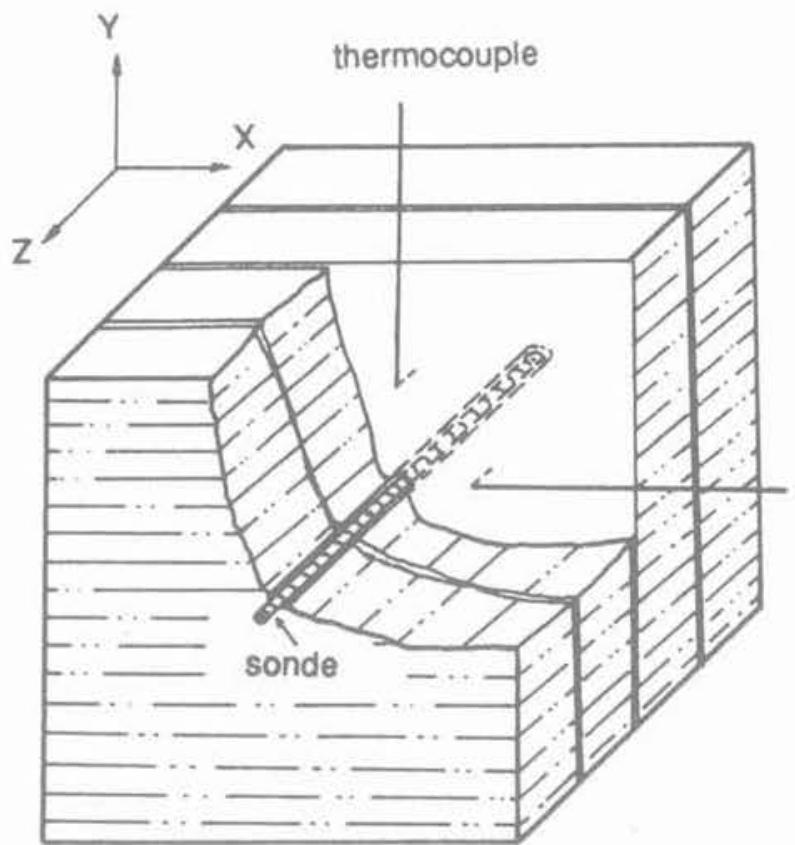

Fig. 2. - Schéma de l'expérience. (lci le bloc est découpé selon 4 plaques). Fig. 2. - View of the sample.

d'acquisition numérique. Compte tenu des caractéristiques de la chaîne, les sorties de température ont une résolution numérique de $+1-0,02^{\circ} \mathrm{C}$.

\section{2. ÉTUDE THÉORIQUE}

\subsection{Position du problème}

Les axes X Y Z coïncident avec les axes principaux de conduction thermique; s'agissant de roches anisotropes (schistes), laxe OZ est contenu dans le plan d'anisotropie majeure (plan de schistosité) et coïncide avec l'axe du fil chaud (fig. 4a). Les isothermes sont donc des cylindres d'axe $\mathrm{OZ}$ dont la directrice est 


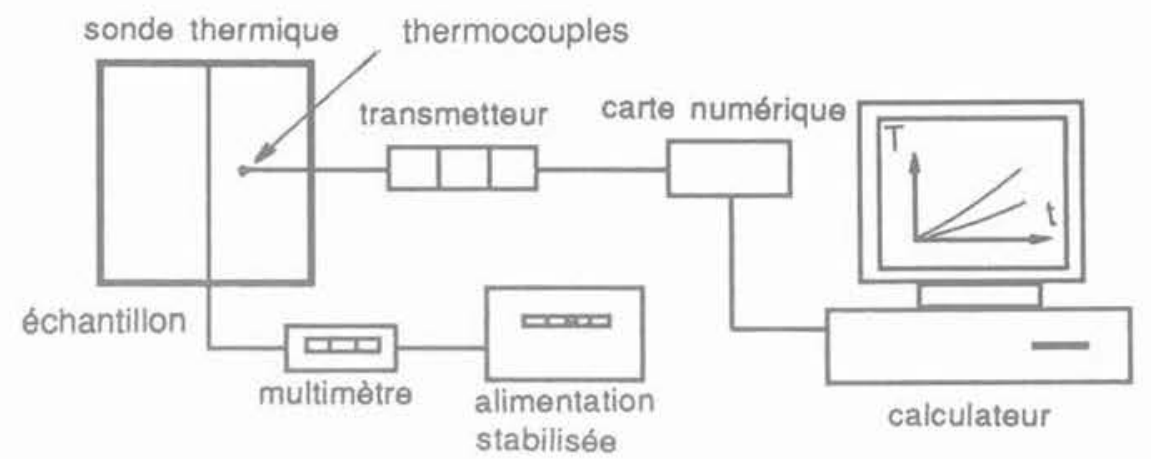

Fig. 3. - Schéma de l'expérimentation et acquisition des données.

Fig. 3. - Scheme of experimentation and data acquisition.
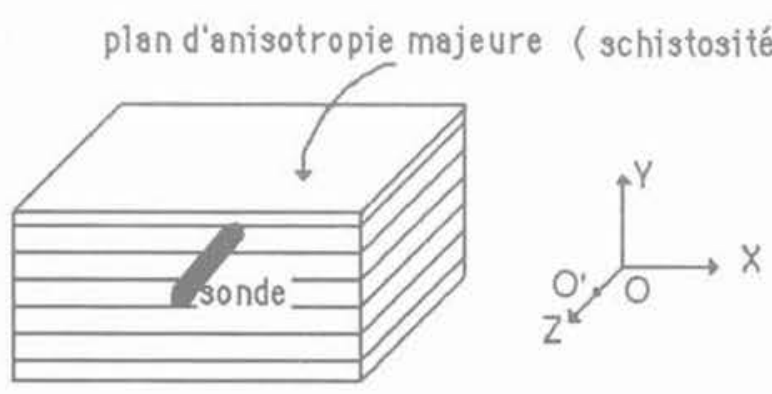

Fig. 4a. - Configuration du problème dans le cas anisotrope. Fig. 4a. - Configuration of the anisotropic problem.

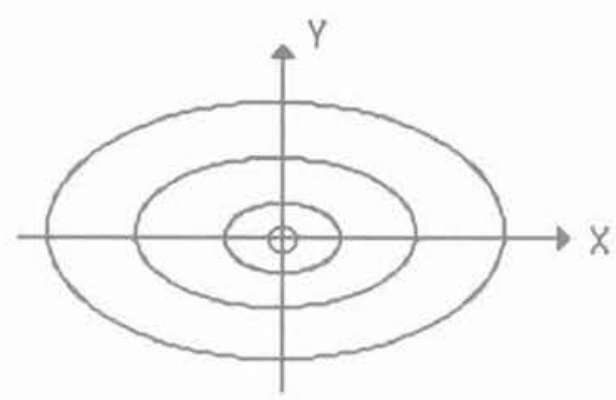

Fig. 4b. - Isothermes dans le plan X-Y.

Fig. $4 b$. - Isotherms in X-Y plane.

Fig. 4. - Configuration du problème.

Fig. 4. - Configuration of the problem

symétrique par rapport aux axes X et $\mathrm{Y}$ (fig. 4b). La résistance chauffante est supposée être un conducteur parfait chauffé à partir de l'instant initial et débitant une puissance constante connue.

Deux hypothèses peuvent être adoptées concernant la sonde:

- sonde infiniment mince (SIM) ;

- sonde cylindrique (SC).

Nous exposons dans ce qui suit, les modèles mathématiques correspondant à ces deux hypothèses.

\subsection{Sonde infiniment mince (SIM)}

CARSLAW et JAEGER (1959) établissent la solution correspondant à une source ponctuelle $\Delta \mathrm{q}$ disposée à l'instant initial au point $O^{\prime}(0,0, Z$ ') de l'axe ? (fig. 4a) :

$$
T(t)=\frac{\Delta q(p C p)^{3 / 2}}{8 \sqrt{\pi^{3} \lambda_{x} \lambda_{y} \lambda_{z} t^{3}}}
$$

$$
\exp \left\{\frac{p C p}{4 t}\left(\frac{X^{2}}{\lambda_{x}}+\frac{Y^{2}}{\lambda_{y}}+\frac{\left(Z-Z^{\prime}\right)^{2}}{\lambda_{z}}\right)\right\}
$$

avec :

T : température du point de coordonnées (X Y Z) ;

$\lambda_{\mathrm{x}}, \lambda_{\mathrm{y}}, \lambda_{\mathrm{z}}$ : conductivités principales, $\mathrm{Wm}^{-1} \mathrm{~K}^{-1}$;

$\mathrm{Cp} \quad$ : chaleur spécifique, $\mathrm{Jkg}^{-1} \mathrm{~K}^{-1}$

$\mathrm{p} \quad$ : masse volumique, $\mathrm{kgm}^{-3}$;

$\Delta \mathrm{q} \quad$ : est défini à partir de la chaleur $\Delta \mathrm{Q}$ placée à l'instant initial en $O$ ', par relation $\Delta \mathrm{q}=\Delta \mathrm{Q} / \mathrm{pCp}$.

Pour une source linéique, infiniment allongée, introduite à l'instant initial (échelon de chaleur), la solution peut être obtenue par intégration par rapport à la coordonnée ' $Z^{\prime}$ et au temps :

$$
T=\frac{q p C p}{4 \pi\left(\lambda_{x} \lambda_{y}\right)^{1 / 2}}
$$

$$
\int_{\frac{p C_{p} r^{2}}{4\left(\lambda_{x} \lambda_{y}\right)^{1 / 2} t}}^{\infty} \frac{e^{-u}}{u} d u
$$

avec : $\quad r^{2}=\sqrt{\frac{\lambda_{y}}{\lambda_{x}}} x^{2}+\sqrt{\frac{\lambda_{x}}{\lambda_{y}}} y^{2}$

Posant: $\quad$ Q $\quad p C p q$ 
et :

$$
-\operatorname{Ei}(-\omega)=\int_{\omega}^{\infty} \frac{e^{-u}}{u} d u
$$

$\mathrm{Ei}$ : fonction exponentielle intégrale ;

Q : quantité de chaleur émise par unité de temps et unité de longueur;

il vient :

$$
T=\frac{-Q}{4 \pi\left(\lambda_{x} \lambda_{y}\right)^{1 / 2}} \text { Ei }\left(-\frac{p C_{p} r^{2}}{4\left(\lambda_{x} \lambda_{y}\right)^{1 / 2} t}\right)
$$

On établit que pour de petites valeurs de $\omega$ :

$$
E i(-\omega)=\gamma+\operatorname{Ln}(\omega)+\sum_{n=1}^{\infty} \frac{(-\omega)^{n}}{n n !}
$$

avec $\gamma$ : constante d'Euler $(\gamma=0,5772)$.

L'équation (4) fournit alors, de manière implicite, les trois paramètres inconnus $\lambda_{\mathrm{x}}, \lambda_{\mathrm{y}}$, et $\mathrm{C}_{\mathrm{p}}$.

Par une méthode d'ajustement, les thermogrammes des points de mesure nous permettent de déterminer ces trois paramètres.

Nous pouvons démontrer que la détermination des trois paramètres nécessite la prise des températures en deux points distincts; une condition supplémentaire garantit l'indépendance des deux mesures (cf. annexe).

La validation de l'hypothèse de sonde infiniment mince dépend de la nature et du rayon $R_{s}$ de la sonde, ainsi que de la résistance thermique son$\mathrm{de} / \mathrm{milieu}$. Dans ce cas le flux thermique à la paroi de la sonde peut être déterminé par dérivation de l'équation (4); par exemple pour le cas isotrope :

$$
\mathrm{K} \frac{\partial \mathrm{T}}{\partial \mathrm{r}}=-\frac{\mathrm{Q}}{2 \pi \mathrm{r}} \mathrm{e}-\frac{\mathrm{r}^{2}}{4 \mathrm{at}}
$$

a : étant la diffusivité thermique.

Dans le cas réel (Rs n'est pas infiniment petit), nous proposons une surestimation des erreurs induites par Phypothèse SIM en supposant que la chaleur spécifique de la résistance chauffante $\left(C_{s}\right)$ est nulle, c'està-dire que la chaleur dégagée par la résistance chauffante est entièrement absorbée par le matériau; le flux thermique au rayon $\mathrm{Rs}$ est alors plus important que dans le cas précédent. La figure 5 illustre les erreurs relatives sur les valeurs des paramètres en fonction du rayon de la perforation. Nous constatons que le rayon doit être inférieur à $1,5 \mathrm{~mm}$ pour garantir une erreur relative inférieure à $5 \%$.

Quand le rayon de la perforation est supérieur à $1,5 \mathrm{~mm}$ nous proposons d'utiliser le modèle de sonde cylindrique ; on introduit alors la dimension de la sonde dans la condition aux limites.

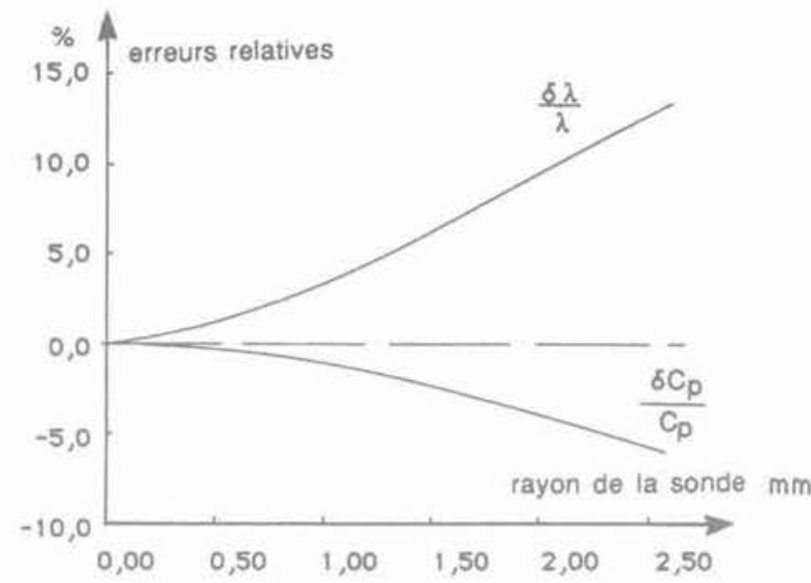

Fig. 5. - Erreurs relatives provoquées par la présence du trou pour la résistance chauffante.

Fig. 5. - Relative errors induced by the presence of the hole for heating resistance.

Données du calcul $r=30 \mathrm{~mm}, a=1 \times 10^{-6} \mathrm{~m}^{2} \mathrm{~s}^{-1}$,
$C_{p}=800 \mathrm{Jg}^{-1} \mathrm{~K}^{-1}, p=2600 \mathrm{~kg} \mathrm{~m}^{-3}, Q=100 \mathrm{Wm}^{-1}$.

\subsection{Sonde cylindrique (SC)}

a. Modèle mathématique dans un milieu isotrope De nombreuses études ont été faites dès les années 50 sur le modèle mathématique de la sonde à choc thermique cylindrique. BLACKWELL (1954) [3], CAESLAW (1959) [5]. En 1956, BLACKWELL [4] publie une étude sur l'erreur que l'on commet quand on néglige le flux de chaleur axial dans la sonde à choc thermique : il établit que le rapport entre la longueur de la sonde et son rayon doit être supérieur à 30 pour que lhypothèse de flux radial soit vérifiée. AUDIBERT (1985) [1] et LAURENT (1986) [11], ont comparé différents outils de sonde à choc thermique cylindrique et fait une optimisation des outils et des méthodes d'identification des caractéristiques thermocinétiques dans les milieux poreux ; les auteurs utilisent deux modèles pour la détermination de la diffusivité et de la chaleur spécifique (fig. 6).

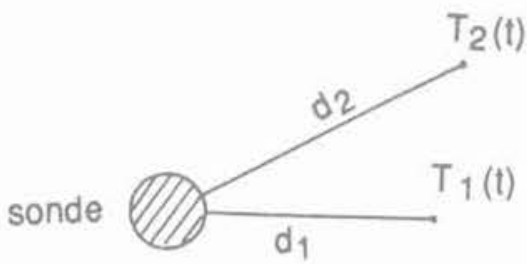

Fig. 6. - Configuration du modèle SC pour un milieu isotrope. Fig. 6. - Configuration of SC model for an isotropic medium.

1. Le modèle dit "température/température ». La température d'un point $\left(\mathrm{d}_{2}\right)$ est modélisée à partir de celle d'un autre point de mesure $\left(\mathrm{d}_{1}\right)$. Les paramètres de flux d'entrée n'interviennent alors plus. Dans un système de coordonnées polaires, on établit que:

$$
\mathrm{T}_{2}^{*}=\int_{0}^{t} \mathrm{~T}_{1}(\tau) \mathrm{H}_{\mathrm{tt}}(\mathrm{t}-\tau) \mathrm{d} \tau
$$


avec :

$$
\begin{gathered}
\mathrm{H}_{\mathrm{tt}}=-\frac{2 \mathrm{a}}{\pi} \int_{0}^{\infty} \exp \left(-\mathrm{au}^{2} \mathrm{t}\right) \\
\frac{\mathrm{J}_{0}\left(\mathrm{ud}_{2}\right) \mathrm{Y}_{0}\left(\mathrm{ud}_{1}\right)-\mathrm{Y}_{0}\left(\mathrm{ud}_{2}\right) \mathrm{J}_{0}\left(\mathrm{ud}_{1}\right)}{\mathrm{J}_{0}^{2}\left(\mathrm{ud}_{1}\right)+\mathrm{Y}_{0}^{2}\left(\mathrm{ud}_{1}\right)} \mathrm{u} \mathrm{d} \mathrm{u}(9)
\end{gathered}
$$

où :

$\mathrm{T}^{*}{ }_{2}$ : température calculée au point $\mathrm{d}_{2}$;

$\mathrm{J}_{0} \mathrm{Y}_{0}$ : fonctions de Bessel de première et de seconde, espèce d'ordre 0 ;

$\mathrm{d}_{1}, \mathrm{~d}_{2}$ : distances entre les points de mesure et la sonde :

a : diffusivité thermique.

Le modèle «température/température * traduisant une relation entre deux grandeurs de même nature (ici deux températures), ne fait donc intervenir qu'un seul paramètre thermique, la diffusivité. Ceci est un avantage pour le processus d'identification.

2. Le modèle dit a flux/température ». La température dans le matériau est modélisée par le flux injecté par la sonde. On établit alors que :

$$
\mathrm{T}^{*}=\int_{0}^{\mathrm{t}} \mathrm{Q}(\tau) \mathrm{H}_{\mathrm{ft}}(\mathrm{t}-\tau) \mathrm{d} \tau
$$

avec :

$$
\begin{aligned}
& \mathrm{H}_{\mathrm{ft}}=-\frac{2}{\pi \mathrm{C}_{\mathrm{p}}} \int_{0}^{\infty} \exp \left(-\mathrm{au}^{2} \mathrm{t}\right) \\
& \frac{J_{0}(\mathrm{ud}) \mathrm{Y}_{1}\left(u R_{\mathrm{s}}\right)-\mathrm{Y}_{0}(\mathrm{ud}) J_{1}\left(u R_{\mathrm{s}}\right)}{J_{1}^{2}\left(u R_{\mathrm{s}}\right)+\mathrm{Y}_{1}^{2}\left(u R_{\mathrm{s}}\right)} \mathrm{du}
\end{aligned}
$$

où :

$J_{1}$ et $Y_{1}$ : fonctions de Bessel de première et de seconde espèce d'ordre un ;

Q : flux thermique émis par unité de surface ; Rs : rayon de la sonde.

Dans la dernière équation les deux paramètres a et $\mathrm{C}_{\mathrm{p}}$ interviennent simultanément.

LAURENT (1986) [11] fournit un processus d'identification des paramètres a et $C_{p}$; l'auteur applique le modèle " température/température " pour calculer a, et le modèle $\propto$ flux/température \& pour calculer $C_{p}$ en utilisant le résultat du modèle précédant. II montre ainsi que la détermination de la diffusivité thermique par modèle « température/température " est indifférente aux conditions de contact thermique sonde/milieu, même si le contact est très mauvais.

\section{b. Adaptation des modèles aux milieux anisotropes}

En reprenant le système de coordonnées cartésiennes, l'équation de la chaleur s'exprime par la relation classique :

$\mathrm{pCp} \frac{\partial \mathrm{T}}{\partial \mathrm{t}}=$

$\lambda\left(\frac{\partial^{2} \mathrm{~T}}{\partial \mathrm{x}^{2}}+\frac{\partial^{2} \mathrm{~T}}{\partial \mathrm{y}^{2}}+\frac{\partial^{2} \mathrm{~T}}{\partial z^{2}}\right)$ en milieu isotrope et :

$\mathrm{pC}_{\mathrm{p}} \frac{\partial \mathrm{T}}{\partial \mathrm{t}}=$

$\lambda_{x} \frac{\partial^{2} T}{\partial x^{2}}+\lambda_{y} \frac{\partial^{2} T}{\partial y^{2}}+\lambda_{z} \frac{\partial^{2} T}{\partial z^{2}}$ en milieu anisotrope avec : $\lambda_{x} \lambda_{y} \lambda_{z}$, conductivités thermiques principales. On peut passer d'un milieu anisotrope à un milieu isotrope, CARSLAW et JAEGER (1959) [5], en effectuant le changement des variables défini par:

$\xi=\sqrt{\frac{\lambda^{\prime}}{\lambda_{x}}} x ; \Psi=\sqrt{\frac{\lambda^{\prime}}{\lambda_{y}}} y ; \zeta=\sqrt{\frac{\lambda^{\prime}}{\lambda_{z}}} z$

où le coefficient $\lambda^{\prime}$ peut être choisi arbitrairement.

L'équation de la chaleur se met alors sous la forme

$\mathrm{pC}_{\mathrm{p}} \frac{\partial \mathrm{T}}{\partial \mathrm{t}}=\lambda^{\prime}\left(\frac{\partial^{2} \mathrm{~T}}{\partial \xi^{2}}+\frac{\partial^{2} \mathrm{~T}}{\partial \Psi^{2}}+\frac{\partial^{2} \mathrm{~T}}{\partial \xi^{2}}\right)$

Dans le nouveau système des variables $\xi \Psi$ et $\zeta$. l'équation de la chaleur (14) est formellement identique à l'équation du milieu isotrope (11). Pour intégrer l'équation, il faut évidemment effectuer le changement de variables sur les conditions aux limites, ce qui risque d'induire quelques difficultés.

Pour le problème du choc thermique cylindrique dans un milieu anisotrope, nous proposons, pour les modèles température/température et flux/température, de poser $\lambda^{\prime}=\sqrt{\lambda_{x}} \lambda_{y}$, (modèle dans le plan $x-y$ ) (fig. 7)

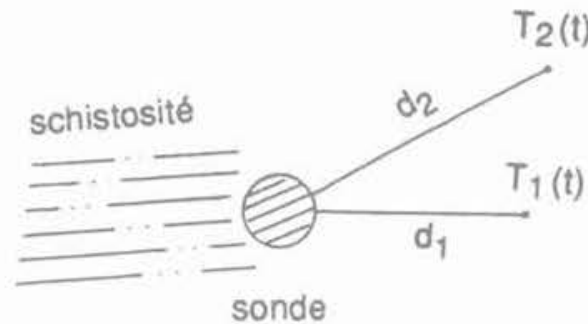

Fig. 7. - Configuration du modèle SC pour un milieu anisotrope.

Fig. 7. - Configuration of SC model for an anisotrope medium.

Les paramètres $a, d_{1}, d_{2}$, Rs dans les modèles $H_{1}$ et $\mathrm{H}_{\mathrm{ft}}$ deviennent alors $\mathrm{a}^{*}, \mathrm{~d}^{*}{ }_{1}, \mathrm{~d}^{*}{ }_{2}, \mathrm{R}^{*}{ }_{5}$ définis par:

$a^{*}=\frac{\sqrt{\lambda_{x} \lambda_{y}}}{p C_{p}} ; \quad d^{*}{ }_{1}=\left(\sqrt{\frac{\lambda_{y}}{\lambda_{x}}} x_{1}^{2}+\sqrt{\frac{\lambda_{x}}{\lambda_{y}}} y_{1}^{2}\right)^{1 / 2}$

$$
d^{*}{ }_{2}=\left(\sqrt{\frac{\lambda_{y}}{\lambda_{x}}} x_{2}^{2}+\sqrt{\frac{\lambda_{x}}{\lambda_{y}}} y_{2}^{2}\right)^{1 / 2} ;
$$$$
R^{*}=\left(\sqrt{\frac{\lambda_{y}}{\lambda_{x}}} x_{s}^{2}+\sqrt{\frac{\lambda_{x}}{\lambda_{y}}} y_{s}^{2}\right)^{1 / 2}
$$ 
où :

$\mathrm{x}_{1} \mathrm{y}_{1}, \mathrm{x}_{2} \mathrm{y}_{2}$ coordonnées des points de mesure $\mathrm{d}_{1}$ et $\mathrm{d}_{2}$

$s_{s}$ et $y_{5} \quad$ définis par $x_{s}{ }^{2}+y_{s}{ }^{2}=R_{s}{ }^{2}$

c. Conditions aux limites et comparaison des modèles «température/température » et «flux/température »

Soulignons que pour que l'hypothèse de la sonde cylindrique soit valable après la transformation des variables (14), le trou de sonde doit être de forme elliptique (fig. 8) défini par la dernière équation $\mathrm{R}^{*}$, ce qui est pratiquement impossible à réaliser techniquement. L'erreur sur cette condition aux limites est d'autant plus faible que le diamètre du trou est petit.

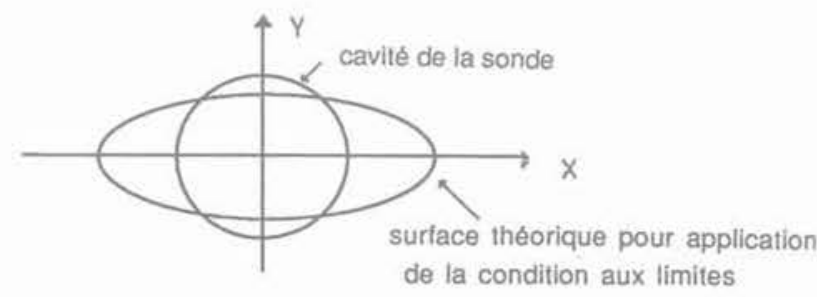

Fig. 8. - Condition aux limites géométriques.

Fig. 8. - Geometrical boundary condition

De plus, par rapport au modèle isotrope la variable $\lambda_{x} / \lambda_{y}$ intervient. Pour le modèle * flux/température * il existe trois variables indépendantes, $\lambda_{x}, \lambda_{y}, C$; mais deux variables seulement, $\lambda_{x} / \lambda_{y}$ et $a^{*}$, pour le modèle * température/température ».

Comme dans le cas isotrope, les variables $\lambda_{x} / \lambda_{y}$ et $\mathrm{a}^{*}$ du modèle * température/température * sont indifférentes aux conditions de contact sonde/milieu. Mais les calculs numériques montrent que ces deux variables sont beaucoup plus sensibles aux erreurs de mesure de température que pour le modèle « flux/température *. Par exemple pour le modèle * température/température * il faut avoir une précision de mesure de $0.005{ }^{\circ} \mathrm{C}$ pour une précision relative des variables de $95 \%$. Par contre pour le modèle «flux/température » il suffit d'une précision de mesure de $0.08^{\circ} \mathrm{C}$ pour atteindre la même précision. En conséquence nous utilisons seulement le modèle "flux/température " pour déterminer les paramètres thermiques de milieux anisotropes.

Cette différence considérable entre les deux modèles s'explique par le fait que le modèle «température/température » élimine les paramètres de flux d'entrée, donc élimine également sa * contribution * pour l'identification des inconnues. Manifestement le flux a la « contribution " la plus importante dans les modèles, parce que c'est lui qui provoque l'évolution de la température.

\subsection{Processus de détermination des paramètres}

La figure 9 présente le processus de détermination des paramètres par le modèle SC ou SIM. Les valeurs initiales des paramètres ne peuvent pas être choisies arbitrairement : il importe de prendre pour ces valeurs initiales, des valeurs réalistes, sinon le processus d'itération peut conduire à des impossibilités (par exemple conductivité négative).

Les modèles SC convergent très lentement (malgré l'élimination des calculs répétés dans la double intégration des modèles) conduisant à des temps de calcul excessifs. Par contre le modèle SIM converge beaucoup plus rapidement. Manifestement quand le diamètre de sonde tend vers zéro, le modèle de SC converge vers le modèle de SIM, ce qui peut servir pour tester les résultats numériques des modèles. La modélisation numérique montre que les paramètres thermiques à déterminer sont moins affectés par l'erreur de mesure de température si les mesures se situent sur les axes principaux de conduction thermique.

Nous utilisons un flux d'entrée de forme échelon. Les thermogrammes aux distances $d_{1}$ et $d_{2}$ (fig. 10) au bout de 150-200 secondes sont utilisés pour déterminer les paramètres thermiques parce que pendant cette période l'effet de bordure latérale ne se fait pas encore sentir. Quant à l'effet de la bordure axiale de l'échantillon, la longueur de la sonde est largement supérieure à 30 fois son diamètre, donc l'hypothèse de sonde infiniment longue reste valable pendant la durée de l'essai.

La qualité de l'ajustement est caractérisée par les valeurs des * résidus * $\left(\mathrm{T}^{*}-\mathrm{T}\right)$. La figure 11 illustre l'évolution des résidus d'un calcul. Ils ont une distribution aléatoire par rapport au temps, dont la moyenne est de l'ordre de $0,015^{\circ} \mathrm{C}$ (qui correspond à la résolution numérique de la mesure de température).

\section{RÉSULTATS EXPÉRIMENTAUX}

\subsection{Paramètres physiques et mécaniques des échantillons}

Cing blocs de roche sont testés, dont quatre schistes et un granite. Le tableau 1 précise leurs paramètres géomécaniques.

Les échantillons sont séchés à $110^{\circ} \mathrm{C}$ pendant 24 heures. Entre deux essais sur un même échantillon, un délai de 10 heures est nécessaire afin d'homogénéiser les températures initiales.

Le choix du modèle SC ou SIM dépend du diamètre de la sonde; s'il est inférieur à 1,5 mm, on utilise le modèle SIM, dans le cas contraire on adopte le modèle SC.

\subsection{Influence de la puissance thermique}

La puissance thermique dégagée par la résistance chauffante a une influence sur la précision relative de 


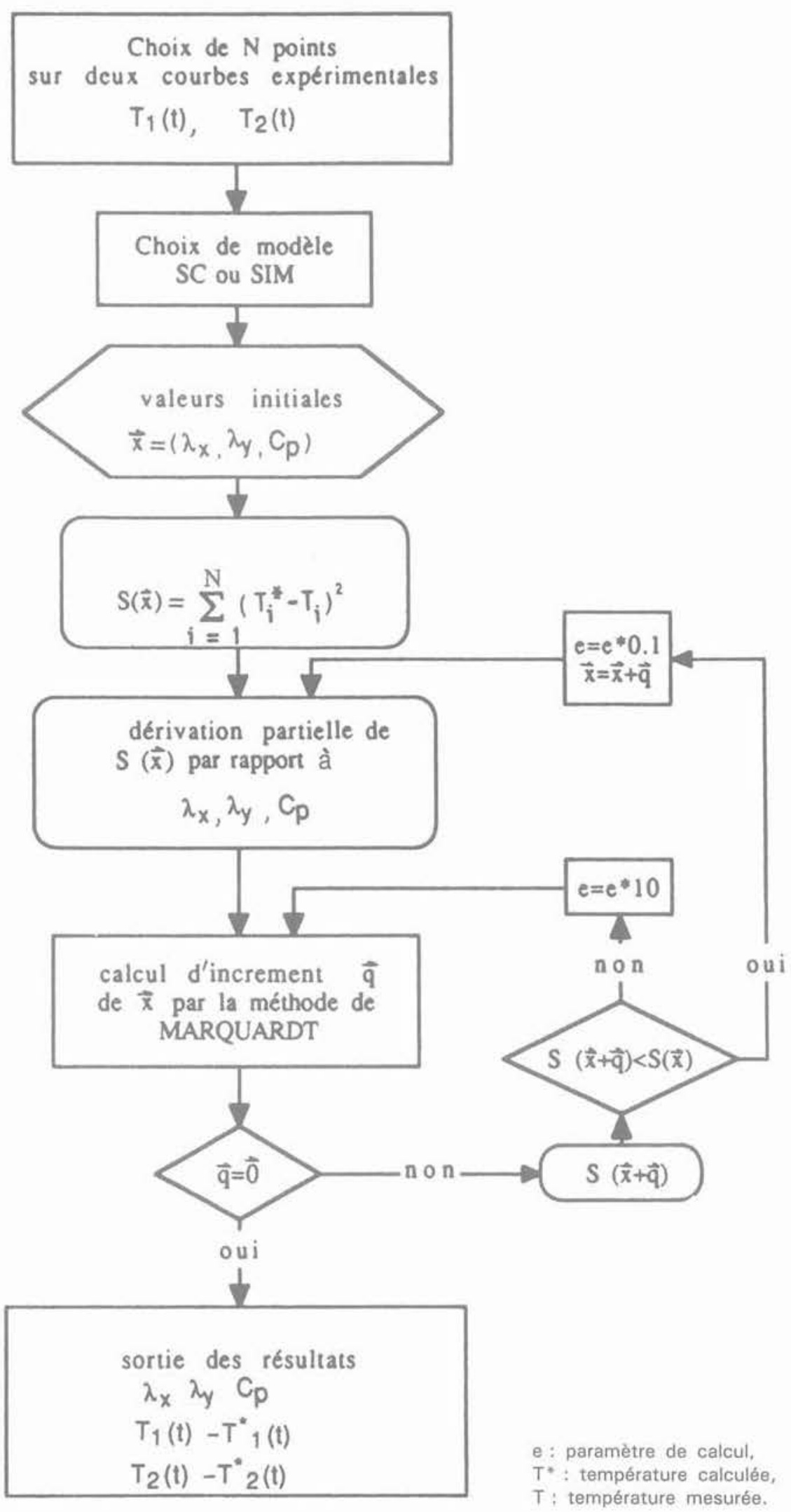

Fig. 9. - Processus d'identification des paramètres thermiques.

Fig. 9. - Identification process for the thermal parameters. 


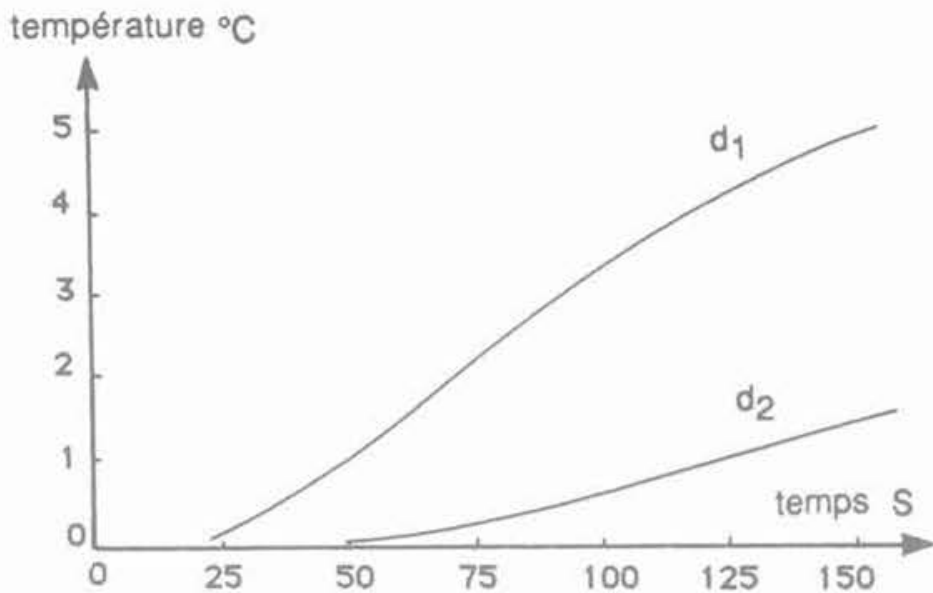

configurgtion de l'essai

$\lambda_{x}>\lambda_{y}$
$d_{1}: X_{1}=25 \mathrm{~mm}, Y_{1}=0$
$d_{2}: X_{2}=0, Y 2=25 \mathrm{~mm}$
$R_{s}=1,5 \mathrm{~mm}$
$Q=302,4 \mathrm{~W} \mathrm{~m}^{-1}$

Fig. 10. - Exemple de thermogrammes expérimentaux.

Fig. 10. - Example of experimental thermograms.

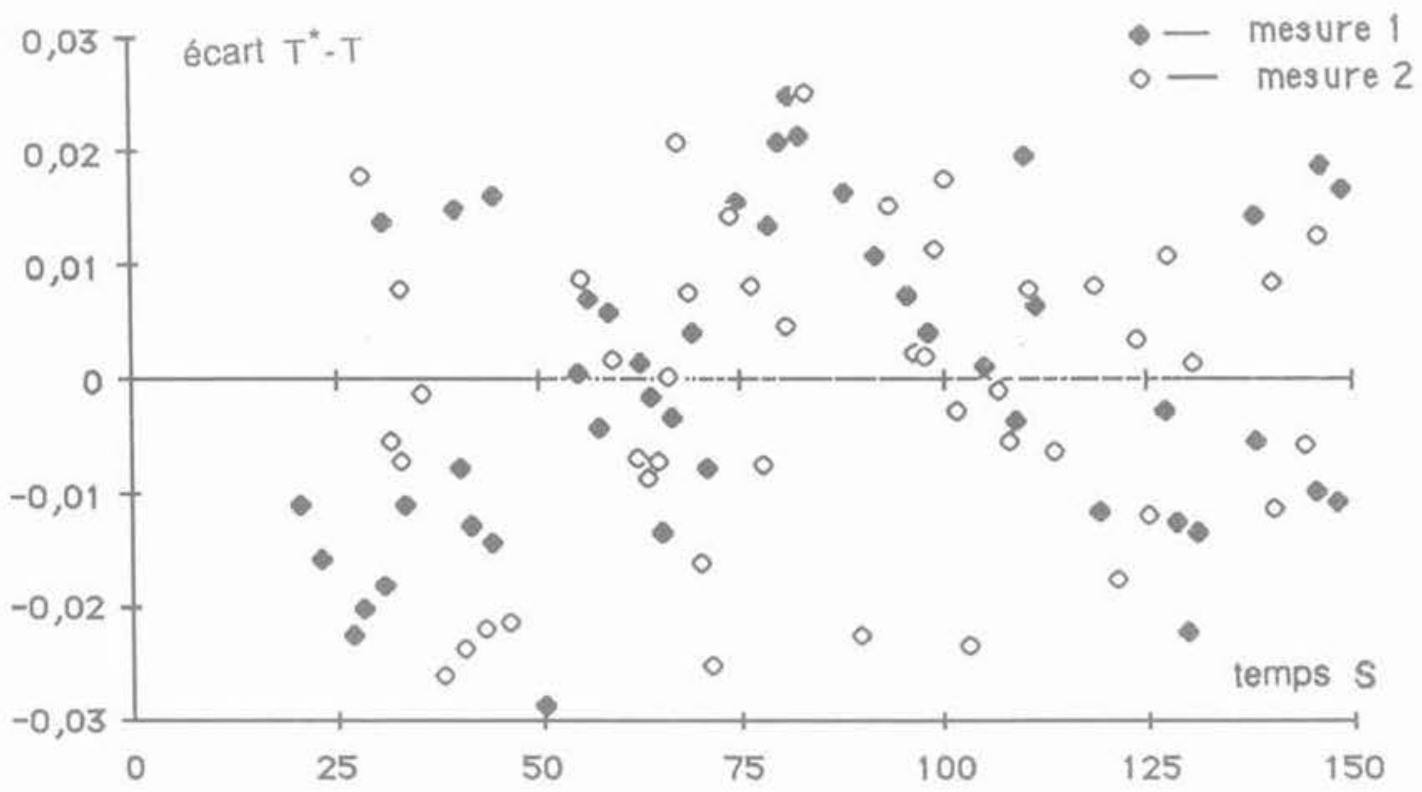

Fig. 11, - Exemple d'évolution des résidus en fonction du temps.

Fig. 11. - Example of residues distribution as a function of time.

Tableau 1. - Paramètres physiques et mécaniques des échantillons Table 1. - Physical and mechanical parameters of the specimens

\begin{tabular}{|c|c|c|c|c|c|c|}
\hline Ech. $n^{\circ}$ & Qualification & $\begin{array}{l}\text { Masse } \\
\text { volumique } \\
\mathrm{kg} \mathrm{m}^{-3}\end{array}$ & $\begin{array}{l}\text { Porosité } \\
\text { totale } \%\end{array}$ & $\begin{array}{c}\text { Célérité } \\
\text { des ondes } \\
\text { longitudinales } \\
\text { (C) } \mathrm{ms}^{-1}\end{array}$ & $\begin{array}{c}\text { Module } \\
\text { d'élasticité } \\
\text { (E) MPa }\end{array}$ & $\begin{array}{l}\text { Résistance } \\
\text { à la compres- } \\
\text { sion simple } \\
\text { (R) (MPa) }\end{array}$ \\
\hline 1 & $\begin{array}{l}\text { Schiste sericito } \\
\text { quartzeux }\end{array}$ & 2650 & 3,0 & $\begin{array}{l}X \quad 6300 \\
Y \quad 4600\end{array}$ & $\begin{array}{l}41300 \\
27600\end{array}$ & $\begin{array}{r}73 \\
140\end{array}$ \\
\hline 2 & $\begin{array}{l}\text { Schiste sericito } \\
\text { quartzeux }\end{array}$ & 2640 & 7,6 & $\begin{array}{ll}X & 6000 \\
Y & 3100\end{array}$ & $\begin{array}{l}29600 \\
27500\end{array}$ & $\begin{array}{r}76 \\
142\end{array}$ \\
\hline 3 & $\begin{array}{l}\text { Gneiss } \\
\text { à amphibolites }\end{array}$ & 2670 & 1,4 & $\begin{array}{ll}X & 5300 \\
Y & 2800\end{array}$ & $\begin{array}{l}34400 \\
31700\end{array}$ & $\begin{array}{l}110 \\
196\end{array}$ \\
\hline 4 & $\begin{array}{l}\text { Schiste } \\
\text { ardoisier }\end{array}$ & 2920 & 2,7 & $\begin{array}{ll}X & 6646 \\
Y & 3500\end{array}$ & $\begin{array}{l}30000 \\
24700\end{array}$ & $\begin{array}{r}68 \\
247\end{array}$ \\
\hline 5 & Monzogranite & 2650 & 1,4 & 4864 & 50000 & 160 \\
\hline
\end{tabular}


la température de mesure. Une série d'essais en fonction de la puissance sont réalisés sur l'échantillon $n^{\circ} 1$ à température ambiante du laboratoire. La figure 12 représente les résultats des calculs pour la détermination de $\lambda_{x}, \lambda_{y}$ et $C_{p}$. Dans la gamme de 1 à 3,5 $W / \mathrm{cm}$ les mesures sont représentatives. En dessous de $1 \mathrm{~W} / \mathrm{cm}$, les mesures de température sont affectées d'une manière importante.

\subsection{Résultats à température ambiante}

Le tableau 2 précise les résultats des essais effectués sur les échantillons à température ambiante (environ $20^{\circ} \mathrm{C}$ ) ; les puissances thermiques sont compri-

Tableau 2, - Résuitats de mesure des paramètres thermiques à température ambiante

Table 2. - Results of mesurement of thermal parameters at ambient temperature

\begin{tabular}{|c|c|c|c|c|c|c|}
\hline \multirow{2}{*}{$\begin{array}{c}\begin{array}{c}\text { Echantil. } \\
n^{0}\end{array} \\
1\end{array}$} & \multicolumn{2}{|c|}{$\underset{m}{W_{m}^{-\lambda_{x}}}{ }^{-1}$} & \multicolumn{2}{|c|}{$\underset{m}{W m_{-1}^{\lambda_{y}}}{ }^{-1}$} & \multicolumn{2}{|c|}{$\begin{array}{c}\mathrm{Cp} \\
\mathrm{J} \mathrm{kg}^{-1} \mathrm{~K}^{-1}\end{array}$} \\
\hline & 4,82 & 0,05 & 2,30 & 0,04 & 821,3 & 17,3 \\
\hline 2 & 3.31 & 0,04 & 1,86 & 0,05 & 709,4 & 19,3 \\
\hline 3 & 3,64 & 0,11 & 1,75 & 0,03 & 848,0 & 16,4 \\
\hline 4 & 4,37 & 0,14 & 1,51 & 0,07 & 787,2 & 11,9 \\
\hline 5 & 3,34 & 0,03 & 3,21 & 0,07 & 834,1 & 17,9 \\
\hline
\end{tabular}

ses entre de 1 et $3 \mathrm{~W} / \mathrm{cm}$. Les résultats sont significatifs au seuil de $2 \%$ à $6 \%(2 \sigma / \mathrm{m})$ avec :

$$
\left\{\begin{array}{l}
\sigma: \text { écart-type } \\
\mathrm{m}: \text { valeur moyenne }
\end{array}\right.
$$

Nous constatons que les anisotropies thermiques sont très marquées pour les schistes. Par exemple, le rapport de la conductivité $\lambda_{x} / \lambda_{y}$ est de l'ordre de 3 pour le schiste ardoisier. Cette anisotropie thermique est évidemment liée à la microstructure de la roche : la condution thermique s'effectue préférentiellement dans le plan de la schistosité où le flux thermique rencontre davantage de "ponts thermiques " (fig. 13).

Il est intéressant de comparer l'anisotropie thermique ̇े celle qui peut être détectée par des essais mécanijues : résistance, module d'Young, célérité des ondes longitudinales (tableau 3). Les anisotropies des différents paramètres ne semblent pas liées.

Tableau 3. - Comparaison du degré d'anisotropie, mesuré à partir des différents paramètres physiques Table 3. - Comparaison of anisotropy of different parameters

\begin{tabular}{|c|c|c|c|c|}
\hline Echantillon & $\frac{\lambda x}{\lambda y}$ & $\frac{E x}{E y}$ & $\frac{R x}{R y}$ & $\frac{C x}{C y}$ \\
\hline 1 & 2,10 & 1,49 & 0,52 & 1,37 \\
\hline 2 & 1,78 & 1,08 & 0,54 & 1,93 \\
\hline 3 & 2,08 & 1,09 & 0,56 & 1,89 \\
\hline 4 & 2,89 & 1,21 & 0,28 & 1,24 \\
\hline
\end{tabular}

conductivité

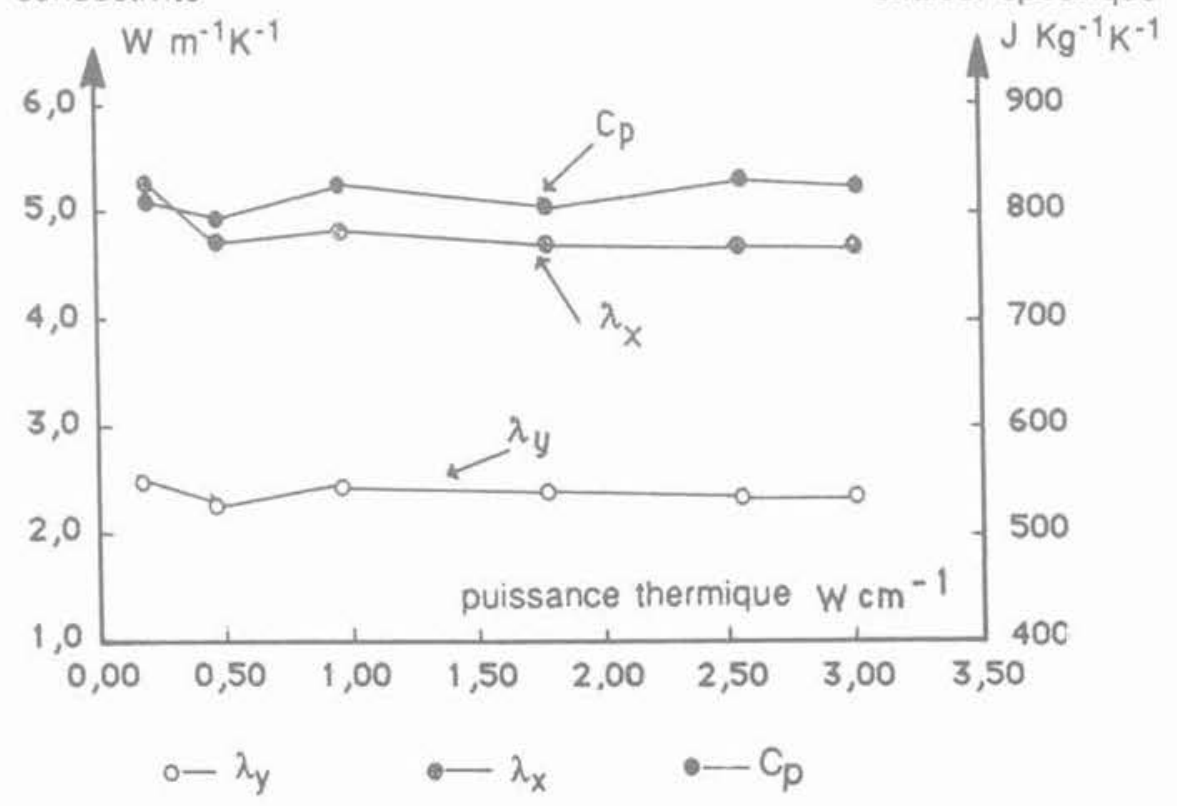

Fig. 12. - Evolution des paramètres thermiques en fonction de la puissance thermique dégagée par la résistance chauffante.

Fig. 12. - Evolution of the thermal parameters with respect to the heat power generated by the heating resistance. 
Fig. 13. - Vue

au microscope électronique

de l'échantillon $n^{\circ} 2$

(schiste sericito-quartzeux).

Fig. 13. - Scanning microscop view of the sample $n^{\circ} 2$

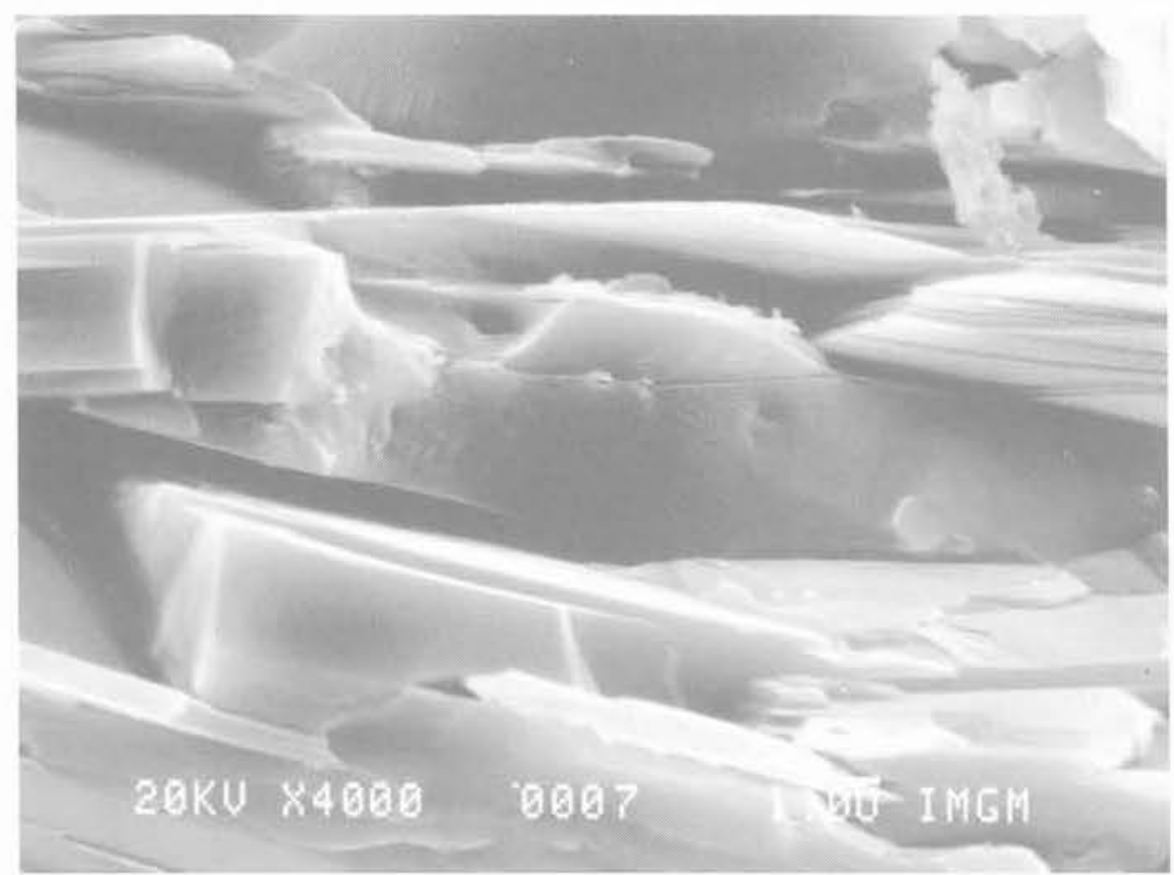

\subsection{Influence de la température ambiante}

La méthode présentée a l'avantage de l'accessibilité de l'échantillon pendant l'essai, ce qui permet d'effectuer des mesures dans différentes conditions de température ambiante (mise en étuve de l'échantillon durant l'essai) ou sous une pression de confinement (essai sous presse).

Nous avons réalisé des essais à diverses températures ambiantes ; de 20 à $250^{\circ} \mathrm{C}$ sur l'échantillon de monzogranite. Pour une température ambiante fixée, l'échantillon est maintenu en étuve pendant 24 heures avant essai.

La figure 14 fournit les conductivités thermiques et la chaleur spécifique du monzogranite en fonction de la température ambiante. On constate que les conductivités diminuent légèrement au fur et à mesure

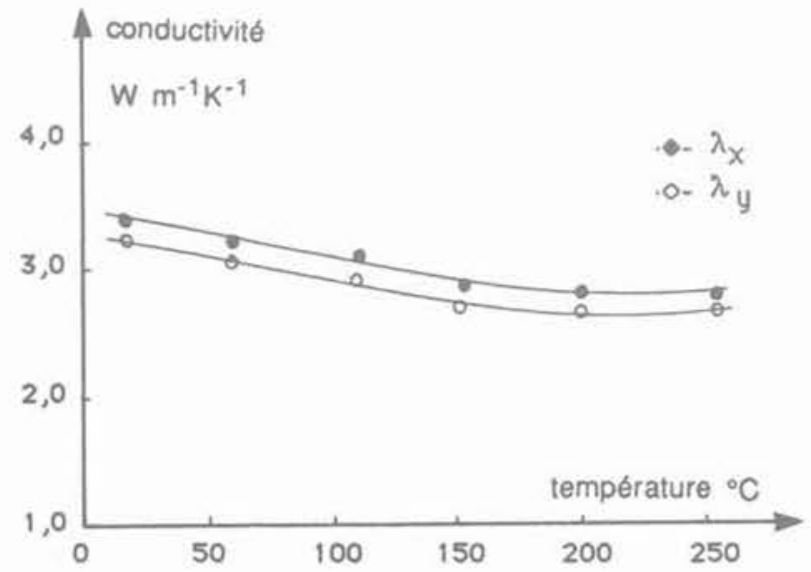

Fig. 14a. - Evolution des conductivités en fonction de la température.

Fig. 14a. - Evolution of conductivities as a function of temperature.

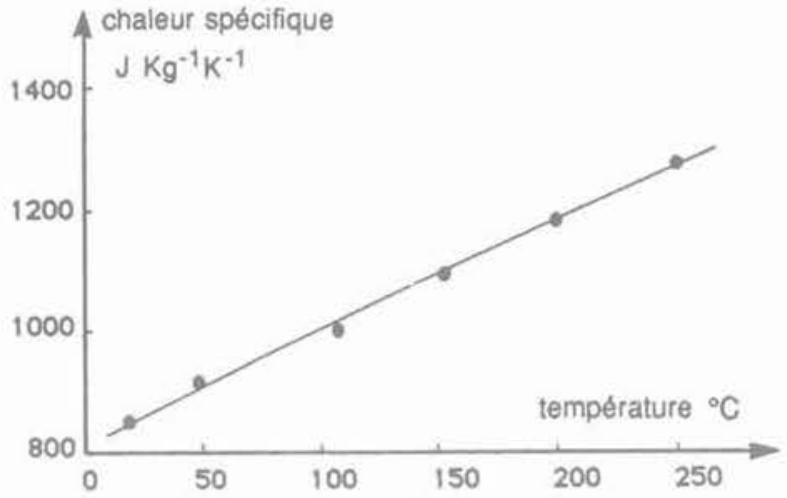

Fig. 14b. - Evolution de la chaleur spécifique en fonction de la température.

Fig. 14b. - Evolution of specific heat a function temperature.

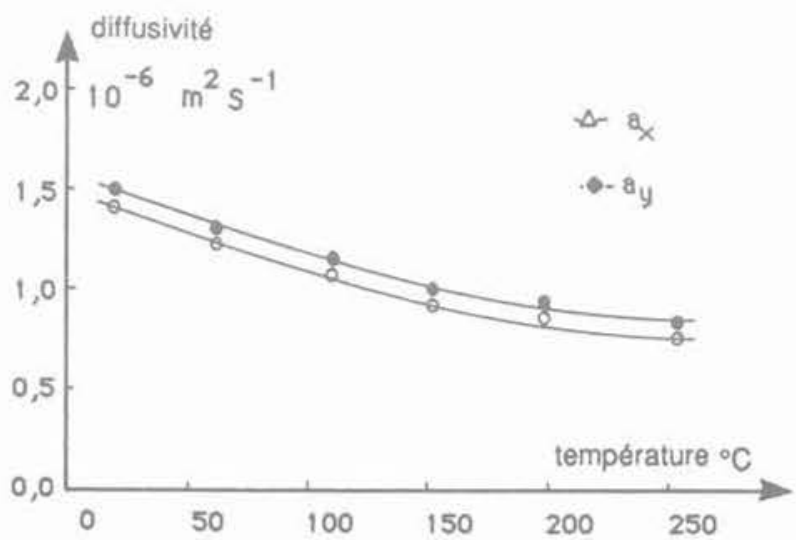

Fig. 14c. - Evolution des diffusivités calculées en fonction de la température.

Fig. $14 c$. - Evolution of diffusivities as a function of temperature,

Fig. 14. - Variation des paramètres $\lambda_{x} \lambda_{y}$ et $C_{p}$ en fonction de la température ambiante de l'échantillon de monzogranite.

Fig. 14. - Variation of thermal parameters $\lambda_{x} \lambda_{y}$ et $C_{p}$ as functions of environment temperature, for monzogranite 
de l'augmentation de la température ambiante (environ de $0,25 \times 10^{-2} \mathrm{Wm}^{-1} \mathrm{~K}^{-1}$ ) : cela peut être expliqué par le développement des microfissurations d'origine thermique. Par contre la chaleur spécifique augmente de façon significative (environ de 1,8 $\mathrm{Jkg}^{-1} \mathrm{~K}^{-1}$ ). Les diffusivités thermiques calculées avec ces deux derniers paramètres diminuent également en fonction de la température, mais c'est l'augmentation de la chaleur spécifique qui joue un rôle prépondérant. La variation de la vitesse des ondes sur les échantillons témoins confirme également la formation de microfissures thermiques en fonction de la température (fig. 15).

\subsection{Influence de propriétés thermiques} non linéaires sur la mesure

Les résultats précédants confirment l'existence de propriétés thermiques non linéaires ; pourtant nous utilisons un modèle théorique linéaire pour déterminer ces paramètres thermiques, L'ajustement des données expérimentales sur un tel modèle risque-t-il de conduire à des valeurs erronées des paramètres thermiques?

La méthode d'ajustement s'effectue à partir des thermogrammes dont l'amplitude est de l'ordre de quelques degrés ; dans cette gamme d'amplitude, la variation des propriétés thermiques apparaît négligeable. Au voisinage de la résistance chauffante, l'écart de température peut atteindre plusieurs dizaines de degrés, mais le volume rocheux est très limité. L'éventualité de non linéarité devrait donc demeurer sans conséquence pour les mesures enregistrées par les thermocouples.

Afin de valider cette remarque, une simulation numérique a été effectuée sur un code de calcul par éléments finis. Les données du calcul numériques sont issues de la configuration correspondant aux essais réalisés. En particulier, les valeurs de $\lambda_{x}, \lambda_{y}$ et $C_{p}$ sont celles des figures $14 \mathrm{a}$ et $14 \mathrm{~b}$. Le tableau 4 présente les températures de la modélisation numérique correspondant au cas non linéaire en fonction du temps. Les écarts de température entre les deux hypothèses de calcul (linéaire et non linéaire) restent

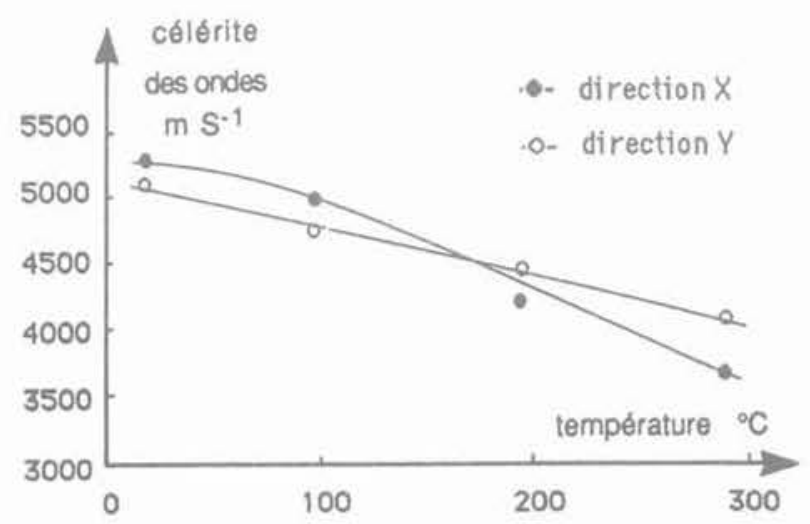

Fig. 15. - Variation de la vitesse des ondes longitudinales en fonction de la température sur les échantillons témoins. Fig. 15. - Variation of longitudinal wave velocity as a function of temperature the vitness specimens.
Tableau 4. - Comparaison des calculs numériques linéaires et non linéaires de propriétés thermiques Table 4. - Comparaison of numerical calculations with linear and non linear thermal properties

\begin{tabular}{|r|c|c|c|c|}
\hline \multirow{2}{*}{$\begin{array}{c}\text { Temps } \\
\text { (S) }\end{array}$} & \multicolumn{4}{|c|}{ Température calculée ${ }^{\circ} \mathrm{C}$} \\
\cline { 2 - 5 } & \multicolumn{2}{|c|}{$\begin{array}{c}\text { Coordonnées } \mathrm{d}_{1} \\
(26,7\end{array}$} & \multicolumn{2}{|c|}{$\begin{array}{c}\text { Coordonnées } \mathrm{d}_{2} \\
(0\end{array}$} \\
\cline { 2 - 5 } & Linéaire & $\begin{array}{c}\text { Non } \\
\text { linéaire }\end{array}$ & Linéaire & $\begin{array}{c}\text { Non } \\
\text { linéaire }\end{array}$ \\
\hline 20 & 0,00625 & 0,00767 & 0,00481 & 0,00602 \\
40 & 0,11314 & 0,11281 & 0,09449 & 0,09454 \\
100 & 1,19199 & 1,18395 & 1,08070 & 1,07019 \\
180 & 2,98248 & 2,97901 & 2,79139 & 2,78834 \\
\hline
\end{tabular}

inférieurs à $10^{-2}{ }^{\circ} \mathrm{C}$, valeur inférieure à la résolution numérique de nos essais.

\section{SYNTHÈSE}

La méthode du choc thermique peut être utilisée pour déterminer les paramètres de conduction thermique des roches anisotropes. Le modèle de choc thermique cylindrique (SC) peut s'adapter aux échantillons où le rayon de la sonde est supérieur à $1,5 \mathrm{~mm}$ pour les roches très dures. Le modèle de sonde infiniment mince (SIM) a l'avantage de permettre l'identification rapide des paramètres, mais le rayon de la sonde devrait être inférieur à $1.5 \mathrm{~mm}$.

Les deux conductivités principales sont mesurées par un essai sur un bloc, ce qui permet d'avoir des résultats représentatifs. Cette méthode permet également d'effectuer des mesures à température élevée et sous pression de confinement.

Les essais effectués sur des roches schisteuses font apparaître une différence importante de conduction thermique entre les directions parallèles et perpendiculaires au plan principal de schistosité. Pour certaines roches à anisotropie très marquée (schistes ardoisiers), le rapport de conductivité peut être de l'ordre de 2,5 à 3 .

Les essais réalisés à des températures ambiantes variant de $20^{\circ} \mathrm{C}$ à $250^{\circ} \mathrm{C}$, font apparaître une non linéarité des propriétés thermiques.

\section{ANNEXE}

Rappel du problème: La détermination des trois paramètres nécessite la prise des températures en deux points distincts $\mathrm{M}_{1}\left(\mathrm{X}_{1}, \mathrm{Y}_{1}\right)$ et $\mathrm{M}_{2}\left(\mathrm{X}_{2}, \mathrm{Y}_{2}\right)$.

Supposons qu'il existe deux solutions distinctes $\left(\lambda_{\mathrm{x}}\right.$, $\left.\lambda_{y}, C_{p}\right)$ et $\left(\lambda^{\prime} x, \lambda_{y}^{\prime}, C_{p}^{\prime}\right)$. Portant ces deux solutions successivement dans l'équation (4), il vient en $\mathrm{M}_{1}$ :

$$
\begin{aligned}
& \lambda_{x} \lambda_{y}=\lambda_{x} \lambda_{y}^{\prime} \\
& C_{p}\left(\lambda_{y} X_{1}{ }^{2}+\lambda_{x} Y_{1}{ }^{2}\right)=C_{p}^{\prime}\left(\lambda_{y} X_{1}{ }^{2}+\lambda_{x}{ }^{\prime} Y_{1}{ }^{2}\right)
\end{aligned}
$$


De même en $\mathrm{M}_{2}$ :

$C_{p}\left(\lambda_{y} X_{2}{ }^{2}+\lambda_{x} Y_{2}{ }^{2}\right)=C_{p}^{\prime}\left(\lambda_{y}^{\prime} X_{2}{ }^{2}+\lambda_{x}^{\prime} Y_{2}{ }^{2}\right)$

(II) et (III), conduisent à :

$\left(X_{1}^{2} Y_{2}^{2}-X_{2}^{2} Y_{1}^{2}\right)\left(\lambda_{x}^{\prime} \lambda_{y}-\lambda_{x} \lambda_{y}^{\prime}\right)=0$

Si $X_{1}^{2} Y_{2}^{2}-X_{2}^{2} Y_{1}^{2} \neq 0$

alors: $\quad \lambda_{x}^{\prime} \lambda_{y}-\lambda_{x} \lambda_{y}^{\prime}=0$

En comparant, cette dernière égalité à (I), nécessairement :

$$
\lambda_{x}^{\prime}=\lambda_{x} ; \lambda_{y}^{\prime}=\lambda_{y} C_{p}^{\prime}=C_{p}
$$

la solution est donc unique.

Si $X_{1}^{2} Y_{2}^{2}-X_{2}^{2} Y_{1}^{2}=0$

c'est-à-dire :

$$
\frac{Y_{1}}{X_{1}}= \pm \frac{Y_{2}}{X_{2}}
$$

la solution n'est pas unique, et elle peut être définie arbitrairement par l'équation (I). La relation (V) décrit les deux droites $D$ et $D^{\prime}$ de la figure ci-dessous.

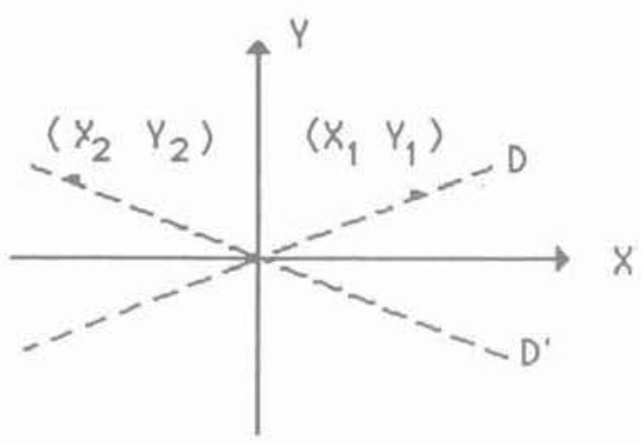

Du point de vue géométrique, en fixant arbitrairement un point $M_{1}\left(X_{1}, Y_{1}\right)$, la dernière équation est une équation de droites symétriques par rapport aux axes. Il apparaît ainsi que les mesures effectuées en deux points quelconques de $\mathrm{D}$ et $\mathrm{D}^{\prime}$ ne sont pas * indépendantes .

\section{BIBLIOGRAPHIE}

[1] AUDIBERT S. (1985), Détermination des caractéristiques thermocinétiques des milieux poreux. Thèse de $3^{e}$ cycle USM/INP Grenoble.
[2] BEREST P., WEBER Ph. (1988), La thermomécanique des roches. Actes de l'Ecole d'été, édition de BRGM n 16.

[3] BLACKWELL J.H. (1954), A transient-flow method for determination of thermal constants of insulating materials in bulk. Canadian Journal of Physics, vol. 25, $n^{\circ} 2, p .137-144$.

[4] BLACKWELL J.H. (1956), The axial-flow error in the thermal-conductivity probe. Canadian Journal of Physics, vol. 34, p. 412-417.

[5] CARSLAW H.S., JAEGER J.C. (1959), Conduction of heat in solids, 2 nd edition. Oxford at the Clarendon Press.

[6] CULL J.P. (1974), Thermal conductivity probes for rapid measurments in rock. Journal of Physics E, Scientific Instrument, vol. 7, p. 771-774.

[7] DESMONS J.Y., MADIETA E., MARTIN M., TORGUET R., LE RAY M. (1984), Nouvelle méthode de mesure de la conductivité thermique. Int. J. Heat Mass Transfer. Vol. 27, $n^{\circ} 4$, p. 511-517.

[8] FOURES J.C., JAVELAS R., PERRIN B. (1981), Caractéristique thermique de matériaux de construction. Détermination. Variation en fonction de la teneur en eau. Revue générale de thermique $n^{\circ} 230$, février 1981.

[9] FOURES J.C., JAVELAS R., PERRIN B. (1980), Application d'une méthode impulsionnelle à la détermination du coefficient de conductivité thermique des matériaux de construction. Revue générale de thermique $n^{\circ} 218$, février 1980.

[10] GROUHEL M.C., GIAT M. (1988), Conductivité thermique apparente de la terre cuite humide non saturée. Revue générale de thermique $n^{\circ} 323$, novembre 1988.

[11] LAURENT (1986), Contribution à la caractérisation thermique des milieux granulaires. Thèse USM/INP, Grenoble.

[12] LEONTIEVA (1985). Théorie des échanges de chaleur et de masse. Traduction française Edition MIR.

[13] PERRIN B., FOURES J.C., JAVELAS R. (1982), Utilisation d'une méthode de chocs thermiques pour la détermination du coefficient de conductivité thermique et de la diffusivité des mortiers et terres cuites. Annuaires de I'ITBTP $n^{\circ}$ 402, février 1982.

[14] QUENARD D., SALLEE H. (1988), Détermination rapide des paramètres thermiques des matériaux par sonde à choc et thermofluxmètres. CSTB Cahier 2295, novembre 1988. 